\title{
Mirogabalin: could it be the next generation gabapentin or pregabalin?
}

\author{
Jae-Yeon $\mathrm{Kim}^{1}$, Salahadin $\mathrm{Abdi}^{2}$, Billy Huh${ }^{2}$, and Kyung-Hoon $\mathrm{Kim}^{1}$ \\ 'Department of Anesthesia and Pain Medicine, Pusan National University School of Medicine, Yangsan, Korea \\ ${ }^{2}$ Department of Pain Medicine, The University of Texas MD Anderson Cancer Center, Houston, TX, USA
}

Received November 30, 2020

Revised December 15, 2020

Accepted December 22, 2020

Handling Editor: Francis S. Nahm

\section{Correspondence}

Kyung-Hoon Kim

Pain Clinic, Pusan National University

Yangsan Hospital, 20 Geumo-ro,

Mulgeum-eup, Yangsan 50612, Korea

Tel: +82-55-360-1422

Fax: +82-55-360-2149

E-mail: pain@pusan.ac.kr
Except for carbamazepine for trigeminal neuralgia, gabapentinoid anticonvulsants have been the standard for the treatment of neuropathic pain. Pregabalin, which followed gabapentin, was developed with the benefit of rapid peak blood concentration and better bioavailability. Mirogabalin besylate (DS-5565, Tarlige ${ }^{\circledR}$ ) shows greater sustained analgesia due to a high affinity to, and slow dissociation from, the $\alpha_{2} \delta$-1 subunits in the dorsal root ganglion (DRG). Additionally, it produces a lower level of central nervous system-specific adverse drug reactions (ADRs), due to a low affinity to, and rapid dissociation from, the $\alpha_{2} \delta$-2 subunits in the cerebellum. Maximum plasma concentration is achieved in less than 1 hour, compared to 1 hour for pregabalin and 3 hours for gabapentin. The plasma protein binding is relatively low, at less than $25 \%$. As with all gabapentinoids, it is also largely excreted via the kidneys in an unchanged form, and so the administration dose should also be adjusted according to renal function. The equianalgesic daily dose for $30 \mathrm{mg}$ of mirogabalin is $600 \mathrm{mg}$ of pregabalin and over $1,200 \mathrm{mg}$ of gabapentin. The initial adult dose starts at $5 \mathrm{mg}$, given orally twice a day, and is gradually increased by $5 \mathrm{mg}$ at an interval of at least a week, to $15 \mathrm{mg}$. In conclusion, mirogabalin is anticipated to be a novel, safe gabapentinoid anticonvulsant with a greater therapeutic effect for neuropathic pain in the DRG and lower ADRs in the cerebellum.

Key Words: Analgesia; Anticonvulsants; Ataxia; Calcium Channels; Cerebellum; Dizziness; Gabapentin; Ganglia, Spinal; Mirogabalin; Neuralgia; Pregabalin; Sleepiness.

\section{INTRODUCTION}

Other than the use of carbamazepine in treating trigeminal neuralgia, gabapentinoids have become the standard drugs in treating neuropathic pain. There are 2 calcium channels in the human body: voltage-gated calcium channels (VGCCs) and ligand-gated (receptor-operated) calcium channels. The mechanism of relief for neuropathic pain is strongly related to the $\alpha_{2} \delta$ ligands which bind to the $\alpha_{2} \delta$ subunits of VGCCs non-specifically.
VGCCs are usually made up of the main pore-forming $\alpha_{1}$ subunit and auxiliary subunits, including the $\beta$ and $\alpha_{2} \delta$, or sometimes $\gamma$ subunits. Four $\alpha_{2} \delta\left(\alpha_{2} \delta-1, \alpha_{2} \delta-2, \alpha_{2} \delta-3\right.$, and $\alpha_{2} \delta$-4) subunit genes have been cloned. The $\alpha_{2} \delta$-1 subunit is widely distributed in the skeletal, smooth, and cardiac muscles, as well as the central and peripheral nervous systems, and endocrine tissues. Cardiac dysfunction or neuropathic pain is the representative pathologic condition with an $\alpha_{2} \delta$-1 subunit disorder. The $\alpha_{2} \delta-2$ subunit is principally located in the central nervous system, especially the (c) This is an open-access article distributed under the terms of the Creative Commons Attribution Non-Commercial License (http://creativecommons.org/licenses/by-nc/4.0/), which permits unrestricted non-commercial use, distribution, and reproduction in any medium, provided the original work is properly cited.

(C) The Korean Pain Society, 2021
Author contributions: Jae-Yeon Kim: Writing/manuscript preparation; Salahadin Abdi: Data curation; Billy Huh: Resources; Kyung-Hoon Kim: Writing/manuscript preparation. 
cerebellum. Pathology in the $\alpha_{2} \delta-2$ subunit may exhibit itself as epilepsy or cerebellar ataxia [1].

Therefore, conventional gabapentinoids, gabapentin and pregabalin, bind to the $\alpha_{2} \delta-1$ and $\alpha_{2} \delta-2$ subunits nonselectively, and produce unwanted adverse drug reactions (ADRs) in the central nervous system, such as dizziness, ataxia, somnolence, and headache. A novel gabapentinoid anticonvulsant needs selectivity for the $\alpha_{2} \delta$ subunits for increasing its therapeutic effect for neuropathic pain via the $\alpha_{2} \delta-1$ subunits, and for decreasing central ADRs through the $\alpha_{2} \delta$-2 subunits.

Mirogabalin besylate (DS-5565, Tarlige ${ }^{\circledR}$; Daiichi Sankyo Company Limited, Tokyo, Japan), which selectively binds to and modulates the $\alpha_{2} \delta$-1 subunits of VGCCs, was recently approved for manufacturing and marketing for treatment of peripheral neuropathic pain in Japan on January 8,2019 , after completing phase 3 clinical trials on patients with diabetic peripheral neuropathic pain (DPNP) and postherpetic neuralgia (PHN). While the drug has received approval for use in Japan, it is still awaiting approval in other countries. Tablets with various doses of mirogabalin besylate are available, including $2.5,5,10$, and $15 \mathrm{mg}$. The initial recommended dose for adults starts from $5 \mathrm{mg}$, given orally twice a day. The dose is slowly increased by 5 $\mathrm{mg}$, at an interval of at least a week, to $15 \mathrm{mg}[2,3]$.

Following studies focusing on selectivity, celecoxib (Celebrex ${ }^{\circledR}$; Pfizer, New York, NY), a selective cyclooxygenase 2 inhibitor non-steroidal antiinflammatory drug (NSAID), or oliceridine (Olinvo ${ }^{\circledR}$; Trevena Inc., King of Prussia, PA), a $\mu$-receptor $\mathrm{G}$ protein pathway selective modulator opioid, has been used or developed for increasing therapeutic effects, while decreasing ADRs [4].

This review examines expectations for mirogabalin, as a novel ligand of the $\alpha_{2} \delta-1$ subunits (compared to the $\alpha_{2} \delta-2$ subunits) containing VGCCs.

\section{MAIN BODY}

\section{VGCCs}

The current trends in development of novel drugs focus on target selectivity, rather than polypharmacy, for achieving increased therapeutic effects, but decreased ADRs [5]. A novel gabapentinoid, mirogabalin, has been introduced for treatment of neuropathic pain, including DPNP and PHN in 2019, highlighting its higher selective binding affinity/slow dissociation half-life to the $\alpha_{2} \delta-1$ rather than the $\alpha_{2} \delta$-2 subunit. However, while, like pregabalin, it showed greater selective binding affinity to the $\alpha_{2} \delta$-1 than to the $\alpha_{2} \delta$-2 subunit, it showed a markedly slower dissociation rate from $\alpha_{2} \delta-1$ compared to $\alpha_{2} \delta-2$, in a human and rat study [6].

Calcium channels can be divided into voltage- and ligand-gated channels. The VGCCs, which open when the membrane potential is changed, include 1) high threshold-activated channels: (1) L [long-lasting or dihydropyridine (DHP), $\mathrm{Ca}_{\mathrm{v}} \mathrm{1} .1$, and 1.2]-, (2) P/Q (Purkinje/question $\mathrm{Ca}_{\mathrm{v}} 2.1$ )-, and (3) N (neural, $\mathrm{Ca}_{\mathrm{v}} 2.2$ )-type, 2) intermediate threshold-activated channels: $\mathrm{Ca}_{\mathrm{v}} 1.3, \mathrm{Ca}_{\mathrm{v}} 1.4$, and $\mathrm{R}$ (residual, $\mathrm{Ca}_{\mathrm{v}}$ 2.3)-type channels, and 3) low thresholdactivated channels: $\mathrm{T}$ (transient, $\mathrm{Ca}_{\mathrm{v}} 3.1,3.2$, and 3.3)-type channels $[7,8]$. However, the ligand-gated calcium channels, which are activated by ligands binding, include the inositol 1,4,5-triphosphate (Ins3P or IP3) receptors, ryanodine receptors, two-pore channels, cation channels of the sperm, and store-operated channels (Table 1) [9].

Among the $\mathrm{Ca}_{\mathrm{v}} 1$ family (L-type channels), $\mathrm{Ca}_{\mathrm{v}} 1.1\left(\alpha_{1} \mathrm{~S}\right.$ subunit) is located in the skeletal muscle, while $\mathrm{Ca}_{\mathrm{v}} 1.2\left(\alpha_{1} \mathrm{C}\right.$ subunit) is found mainly in the cardiac muscle and neurons. $\mathrm{Ca}_{\mathrm{v}} 1.3\left(\alpha_{1} \mathrm{D}\right.$ subunit) has a role in neurotransmission in auditory cells and pacemaker activity, and $\mathrm{Ca}_{\mathrm{v}} \mathrm{l} .4\left(\alpha_{1} \mathrm{~F}\right.$ subunit) acts on synaptic transmission in the retina $[7,8]$.

The VGCCs contain 5 different subunits: $\alpha_{1}(170-240$ $\mathrm{kDa}), \alpha_{2}(150 \mathrm{kDa}), \beta(50-78 \mathrm{kDa}), \delta(17-25 \mathrm{kDa})$, and $\gamma(32$ $\mathrm{kDa})$ in stoichiometric amounts $[7,8,10]$. The main poreforming $\alpha_{1}$ subunit is bonded non-covalently to the auxiliary $\alpha_{2}, \beta$, and $\delta$ subunits, as well as to calmodulin, which modulates the calcium ion trafficking and biophysical properties of the main $\alpha_{1}$ subunit $[9,10]$.

\section{1) Main pore-forming $\alpha_{1}$ subunits of VGCCs}

The pore-forming $\alpha_{1}$ subunit has 24 transmembrane $\alpha$-helices, making 4 homogenous repeats (I-IV). The 4 th transmembrane segment (S4) of each repeat has 5 positively charged amino acids with the 1st, 2nd, and 3rd segments (S1, S2, and S3) producing the voltage-sensing domain of the channel. There are pore loops present between the 5th and 6th segments (S5 and S6). There are also loops between I (S6) and II (S1), II (S6) and III (S1), and III (S6) and IV (S1) (Fig. 1) [9,11].

\section{2) Auxiliary $\alpha_{2}, \beta$, and $\delta$ subunits for modulating $\alpha_{1}$ subunits of VGCCs}

The $\alpha_{2}, \delta$, and $\beta$ subunits are located extracellularly, in the membrane, and intracellularly, respectively.

\section{(1) $\beta$ subunits}

The intracellular (cytosolic) $\beta$ subunits are composed of an Src homology $\left(\mathrm{SH}_{3}\right)$ domain and a guanylate kinase (GK) domain. The GK domain binds to the intracellular linker 


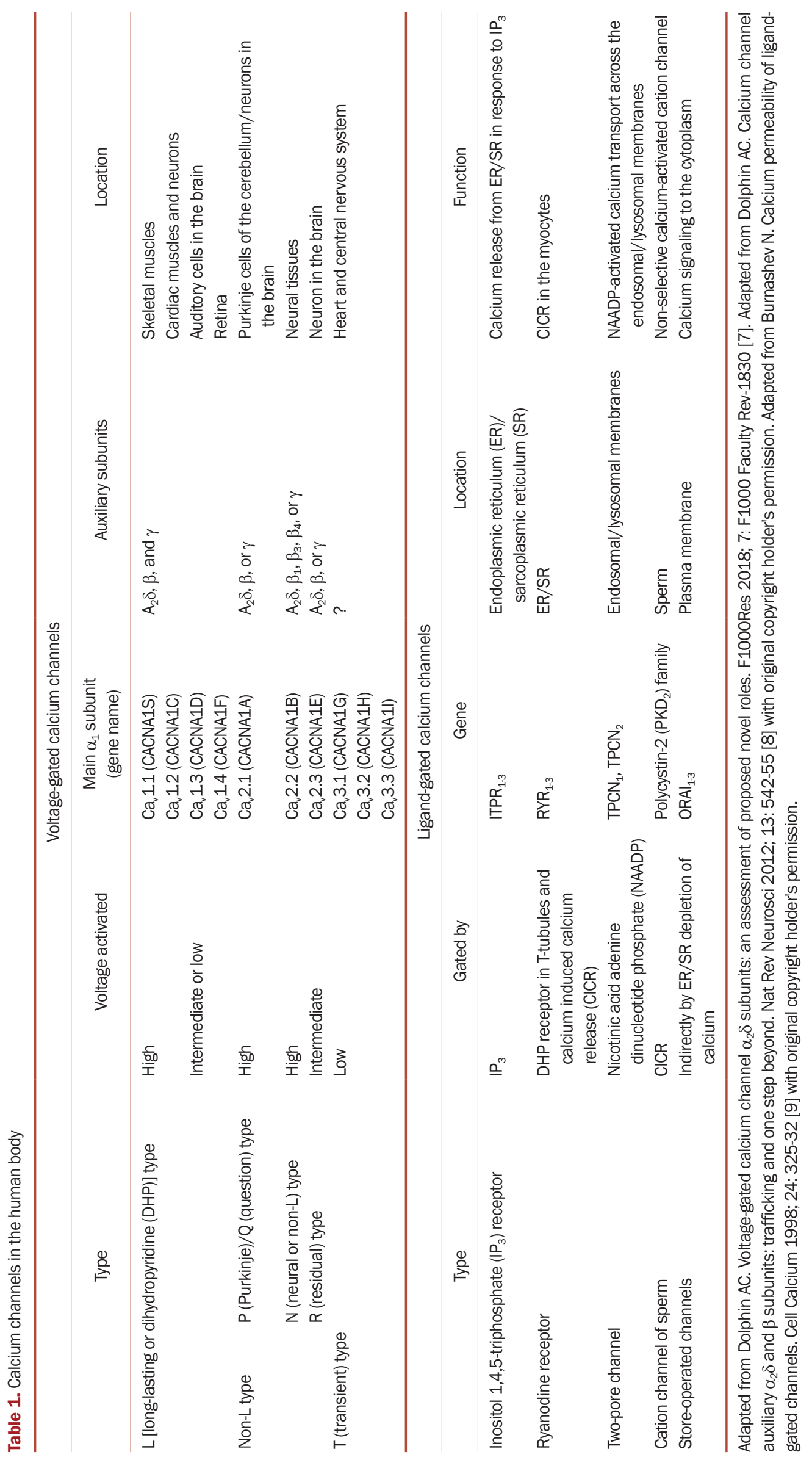




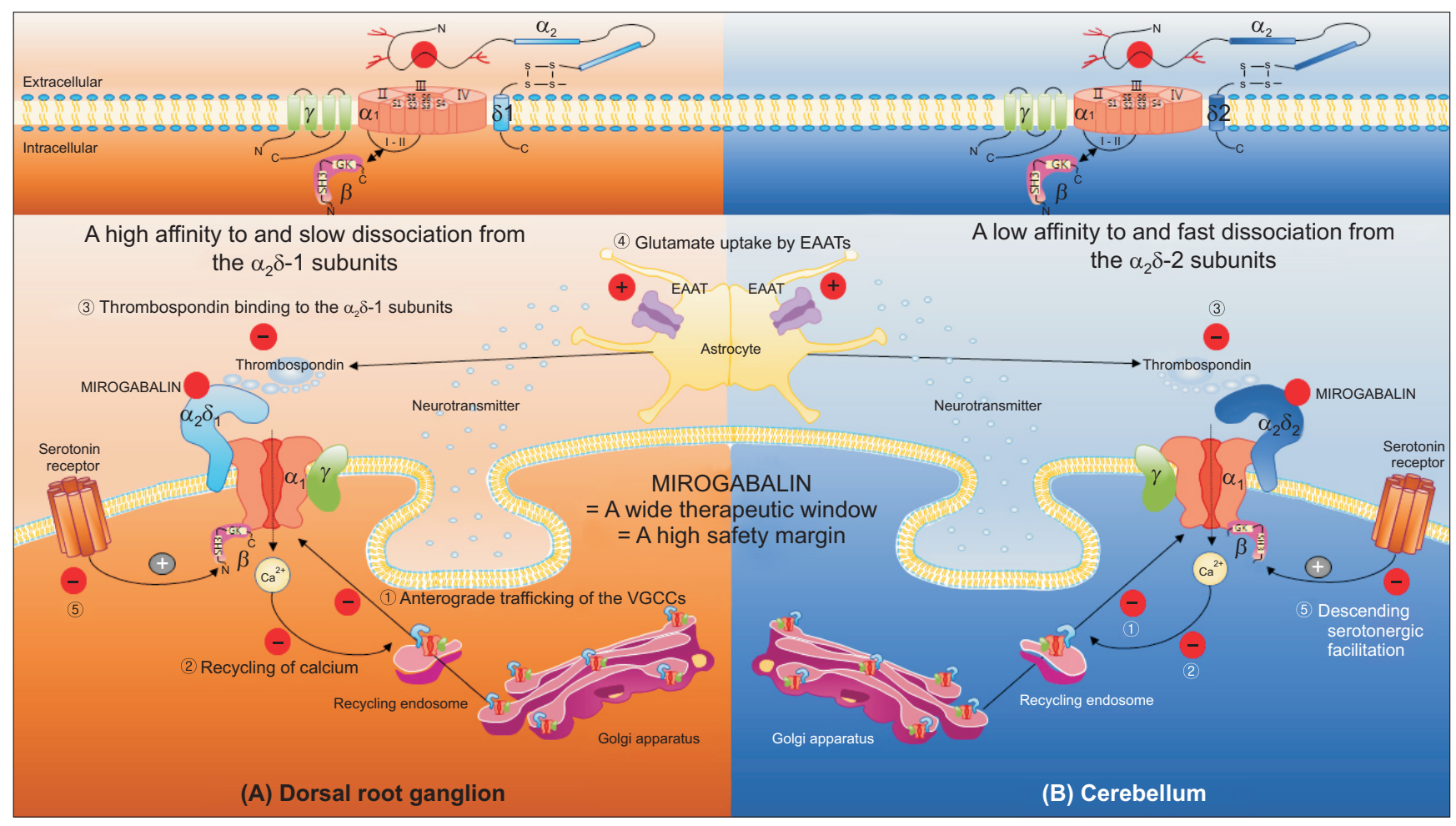

Fig. 1. A schematic illustration of the mechanisms of action for mirogabalin. Mirogabalin has a high affinity to and slow dissociation from the $\alpha_{2} \delta-1$ subunits in the dorsal root ganglia (DRGs), producing greater therapeutic effects; it also has a low affinity to and fast dissociation from the $\alpha_{2} \delta-2$ subunits in the cerebellum, producing lesser adverse drug reactions. In general, various mechanisms of action for gabapentinoids are suggested from inside the cell, membrane, and synapse. (1) Gabapentinoids inhibit forward (anterograde) trafficking of voltage-gated calcium channels (VGCCs) (from the endoplasmic reticulum through the Golgi complex to the cell membrane) intracellularly. Inhibition of anterograde trafficking reduces VGCCs, calcium entry, and excitatory amino acids (glutamates). (2) They inhibit the Rab-11-dependent final recycling of endosomal VGCCs intracellularly, resulting in reduced excitatory neurotransmitter release in the synapse. The small guanosine triphosphatases (GTPases, Rab) are the main regulators of intracellular membrane trafficking, from formation of transport vesicles to their fusion into the membranes. Reduced recycling of endosomal VGCCs results in reduced transmembrane VGCCs, calcium entry into the cell, and glutamate in the synapse. (3) They inhibit astrocyte-derived thrombospondin (TSP, extracellular matrix protein)-mediated excitatory synapse formation extracellularly (reduction of excitatory synaptogenesis). (4) They stimulate glutamate uptake by excitatory amino acid transporters (EAATs) extracellularly. (5) Gabapentinoids may show inhibition of descending serotonergic facilitation, stimulation of descending inhibition, anti-inflammatory effect, and influence on the affective component of pain. A magnified illustration for the transmembrane VGCC is shown in the upper left (in the DRGs) and right (in the cerebellum) quadrants. Adapted from Chincholkar M. Analgesic mechanisms of gabapentinoids and effects in experimental pain models: a narrative review. Br J Anaesth 2018; 120: 1315-34 [14].

between domains I and II of the $\alpha_{1}$ subunit. The membrane-anchored $\alpha_{2} \delta$ subunit consists of the extracellular $\alpha_{2}$ and membrane-associated $\delta$ subunits. They are connected with a disulfide bond (Fig. 1) [9,11].

\section{(2) $\alpha_{2} \delta$ subunits}

The membrane (glycosylphosphatidylinositol, GPI)anchored $\alpha_{2} \delta$ subunit is tasked with modulation of the calcium channel current kinetics, and with increasing trafficking of the channel to the membrane. The $\alpha_{2} \delta$ subunit determines VGCC abundance in the presynaptic terminals, and configures synaptic VGCCs to drive exocytosis through an extracellular metal ion-dependent adhesion site (MIDAS), a conserved set of amino acids with the predicted von Willebrand A (VMA) domain of the $\alpha_{2} \delta$ subunit [12].
At least $4 \alpha_{2} \delta$ subunit genes are cloned: (1) The $\alpha_{2} \delta-1$ subunit mRNA was cloned from skeletal muscle $\left(\mathrm{Ca}_{\mathrm{v}} \mathrm{l} .1\right)$; however, it is found throughout the human body, including the cardiac and smooth muscles associated with $\mathrm{Ca}_{\mathrm{v}} 1.2$. It is also found in many neuronal cell types and the dorsal root ganglia (DRGs), especially in excitatory rather than inhibitory interneurons. (2) The $\alpha_{2} \delta-2$ subunit mRNA is found mainly in the brain (such as the Purkinje cells in the cerebellum, medulla, hippocampus, and striatum) or in the lungs. (3) The $\alpha_{2} \delta-3$ subunit mRNA is found in the brain (cerebral cortex, caudate-putamen, and hippocampus), heart, and skeletal muscle. (4) The recently identified $\alpha_{2} \delta-4$ subunits are distributed in the non-neuronal tissues, such as the adrenal or pituitary glands. The human $\alpha_{2} \delta-4$ protein sequence shares $30 \%, 32 \%$, and $61 \%$ of its identity with human $\alpha_{2} \delta-1, \alpha_{2} \delta-2$, and $\alpha_{2} \delta-3$, respectively. The $\alpha_{2} \delta-4$ subunits are located in the retina, therefore, they are deeply 
related to night blindness $[1,13]$.

\section{Action mechanisms of mirogabalin}

\section{1) Conventional gabapentinoids}

Gabapentin and pregabalin bind to the $\alpha_{2} \delta-1$ and $\alpha_{2} \delta$ 2 subunits, especially, to the 3 rd arginine ( $R$, rectus) in the RRR motif, which is located N-terminal to the VMA domain. The $\alpha_{2} \delta$-1 subunit plays a role in development of chronic neuropathic pain, and is the target for treatment. The $\alpha_{2} \delta-2$ subunit is concentrated in the Purkinje cells of the cerebellum, and this explains why the conventional gabapentinoid anticonvulsants present the representative ADRs, such as dizziness and ataxia (Fig. 1) [1].

In general, mechanisms of action for gabapentinoids include: (1) Gabapentinoids inhibit forward (anterograde) trafficking of $\alpha_{2} \delta-1$ (from the endoplasmic reticulum through the Golgi complex to the cell membrane) intracellularly. (2) They inhibit Rab-11-dependent final recycling of endosomal VGCCs intracellularly, resulting in reduced excitatory neurotransmitter release in the synapse. (3) They inhibit thrombospondin (TSP, extracellular matrix protein)-mediated processes extracellularly, resulting in reducing excitatory synaptogenesis. (4) They stimulate glutamate uptake by excitatory amino acid transporters extracellularly. (5) Minor mechanisms related to gabapentinoids may include inhibition of descending serotonergic facilitation, stimulation of descending inhibition, antiinflammatory effect, and influence on the affective component of pain [14] (Fig. 1).

The $\alpha_{2} \delta-1$ and $\beta$ subunits in the DRGs mediate forward trafficking of calcium channels (exocytosis) to the dorsal horn from the endoplasmic reticulum which is facilitated by protein kinase $\mathrm{C}$ (PKC) through the Golgi complex to the cell membrane [15].

Gabapentinoids act on the DRGs and A nerve fibers, especially medium-sized neurons associated with $A \delta$ nerve fibers and small isolectin B4 (IB4)-negative DRGs, projecting to the excitatory neurons in the lamina I and II of the spinal dorsal horn (compared to large neurons associated with $\mathrm{A} \beta$ nerve fibers and IB4-positive neurons, projecting to the inhibitory neurons) [14].

The $\alpha_{2} \delta-1$ and $\beta$ subunits of the VGCCs mediate forward trafficking of the calcium channels from the endoplasmic reticulum, facilitated by PKC. The reduced recycling of endosomal VGCCs leads to a reduced calcium channel expression and decreased transmitter release at the synaptic membrane. Gabapentinoids inhibit Rab-11 (master regulators of the surface expression of receptors and adhesion protein)-dependent recycling of endosomal VGCCs. In addition, TSP 4 (a family of 5 extracellular matrix oligomeric glycoproteins) mediates excitatory synaptogenesis with cellular migration, attachment, and cytoskeletal dynamics. It also mediates the binding to the $\alpha_{2} \delta-1$ subunits. However, gabapentin does not seem to target TSPs $/ \alpha_{2} \delta-1$ directly $[15,16]$.

On the other hand, 2 systems for intracellular calcium extrusion include the plasma membrane $\mathrm{Ca}^{2+}$ ATPase (PMCA) and plasma membrane $\mathrm{Na}^{+} / \mathrm{Ca}^{2+}$ exchanger (NCX). The NCX has a low calcium affinity but a high capacity for calcium transport, whereas the PMCA has the opposite properties [17].

2) Comparison of binding and dissociation kinetics of gabapentin, pregabalin, and mirogabalin for the $\alpha_{2} \delta-1$ and $\alpha_{2} \delta$-2 subunits

Gabapentinoids, including gabapentin and pregabalin, have become the drugs of choice for the treatment of neuropathic pain with positive symptoms [4].

Binding affinity is measured by the equilibrium dissociation constant $\left(\mathrm{K}_{\mathrm{d}}\right)$. The smaller the $\mathrm{K}_{\mathrm{d}}$ value, the greater the binding affinity of the ligand for its target. Gabapentin is known to bind to $\alpha_{2} \delta$ subunits with greater affinity for $\alpha_{2} \delta-1\left(K_{d}=59 \mathrm{nmol} / \mathrm{L}\right)$ compared to $\alpha_{2} \delta-2\left(K_{d}=153 \mathrm{nmol} / \mathrm{L}\right)$ [15].

Pregabalin also shows greater affinity to the $\alpha_{2} \delta-1\left(K_{d}=\right.$ $62.5 \mathrm{nmol} / \mathrm{L})$ compared to the $\alpha_{2} \delta-2\left(\mathrm{~K}_{\mathrm{d}}=125.0 \mathrm{nmol} / \mathrm{L}\right)$. Mirogabalin similarly exhibits greater affinity to the $\alpha_{2} \delta-1$ $\left(K_{d}=13.5 \mathrm{nmol} / \mathrm{L}\right)$, compared to the $\alpha_{2} \delta-2\left(K_{d}=22.7 \mathrm{nmol} /\right.$ L). However, pregabalin show similar dissociation time from the $\alpha_{2} \delta-1\left[\mathrm{~K}_{\text {off }}=0.5051\right.$ per hour] and the $\alpha_{2} \delta-2\left[\mathrm{~K}_{\text {off }}=\right.$ 0.5103 per hour]. Mirogabalin exhibits slow dissociation from the $\alpha_{2} \delta-1$ [K $\mathrm{K}_{\text {off }}=0.0627$ per hour] compared to the $\alpha_{2} \delta$ $2\left[\mathrm{~K}_{\text {off }}=0.2837\right.$ per hour]. Its dissociation half-life is also longer from the $\alpha_{2} \delta-1\left(\mathrm{t}_{1 / 2}=11.1\right.$ per hour) than the $\alpha_{2} \delta-2\left(\mathrm{t}_{1 / 2}\right.$ $=2.4$ per hour) [6].

Therefore, mirogabalin, compared with pregabalin, has a similar degree of greater affinity to the $\alpha_{2} \delta-1$ compared to the $\alpha_{2} \delta-2$, however, its dissociation time from the $\alpha_{2} \delta-1$, compared to the $\alpha_{2} \delta-2$, is longer than with pregabalin. The secret is that mirogabalin, compared to pregabalin, shows a greater therapeutic analgesic effect for neuropathic pain and lesser ADRs due to the slow dissociation from the $\alpha_{2} \delta$ 1 subunits, not due to the binding affinity to the $\alpha_{2} \delta-1$ subunits. Mirogabalin has a longer dissociation half-life from the $\alpha_{2} \delta-1$ subunits than the $\alpha_{2} \delta-2$ subunits, in contrast to pregabalin.

In conclusion, mirogabalin has a high affinity to and slow dissociation from the $\alpha_{2} \delta$-1 subunits in the DRGs, producing greater therapeutic effects; it also has a low affinity to and fast dissociation from the $\alpha_{2} \delta-2$ subunits in the cerebellum, producing lesser ADRs. 


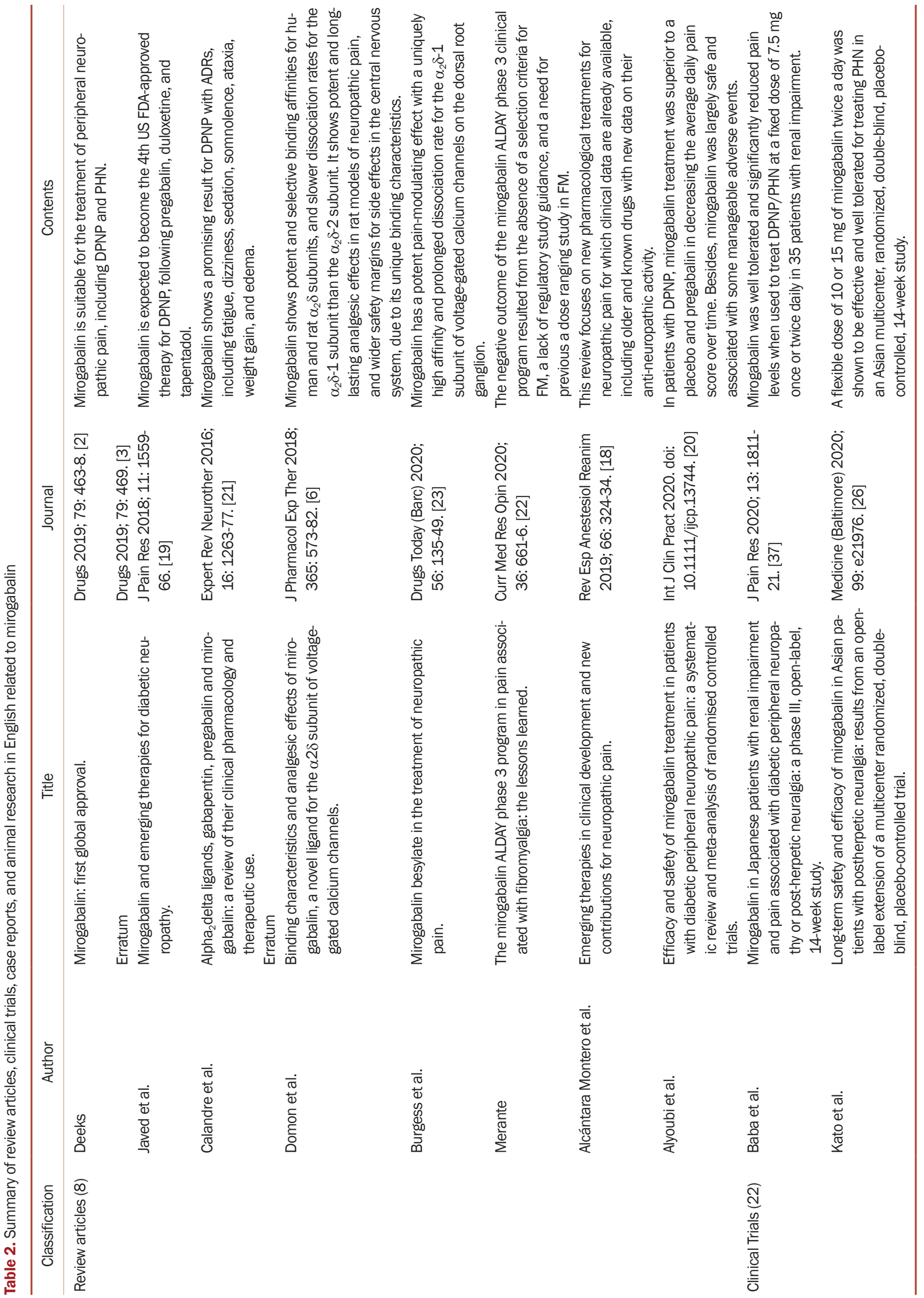



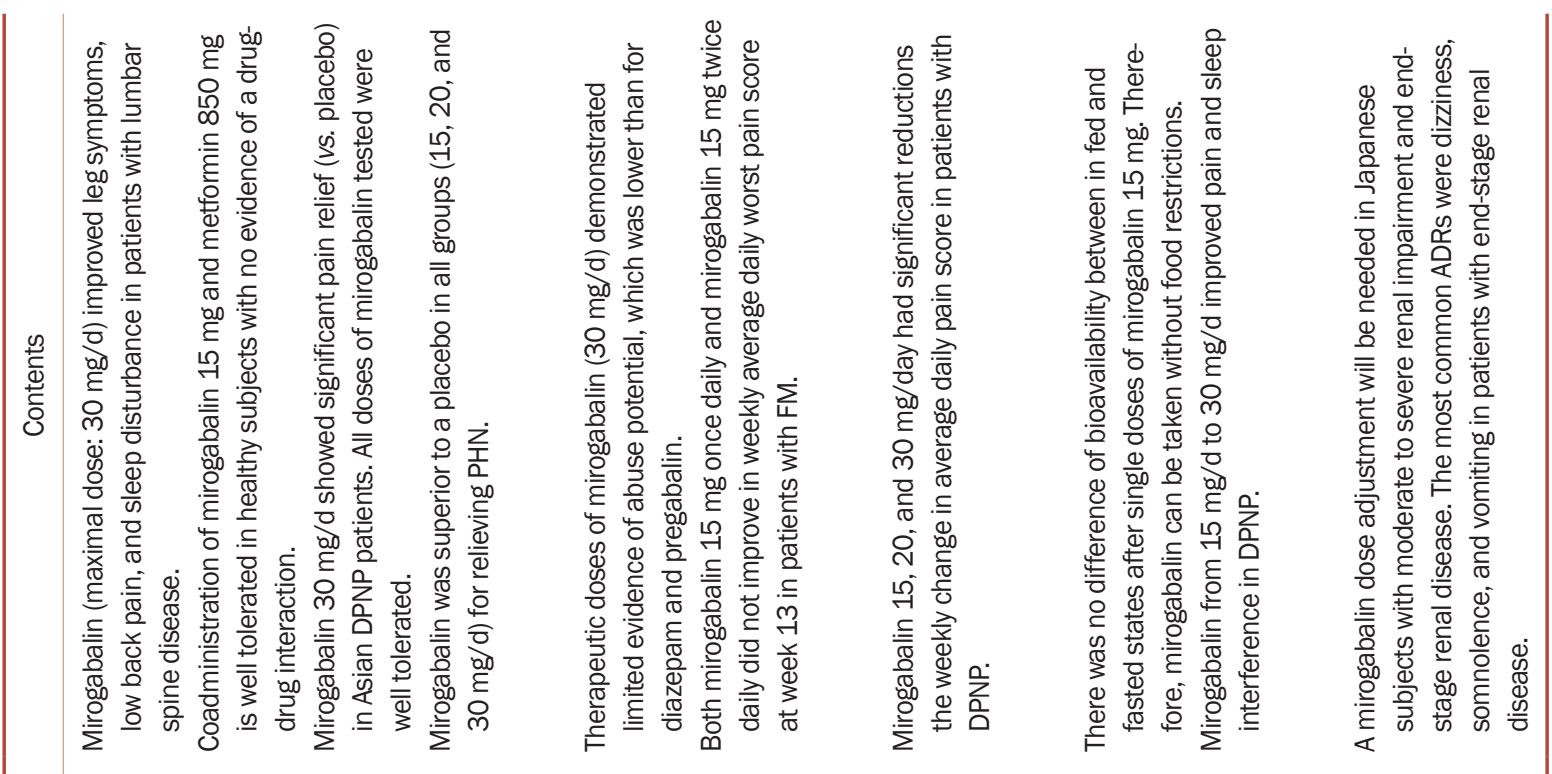

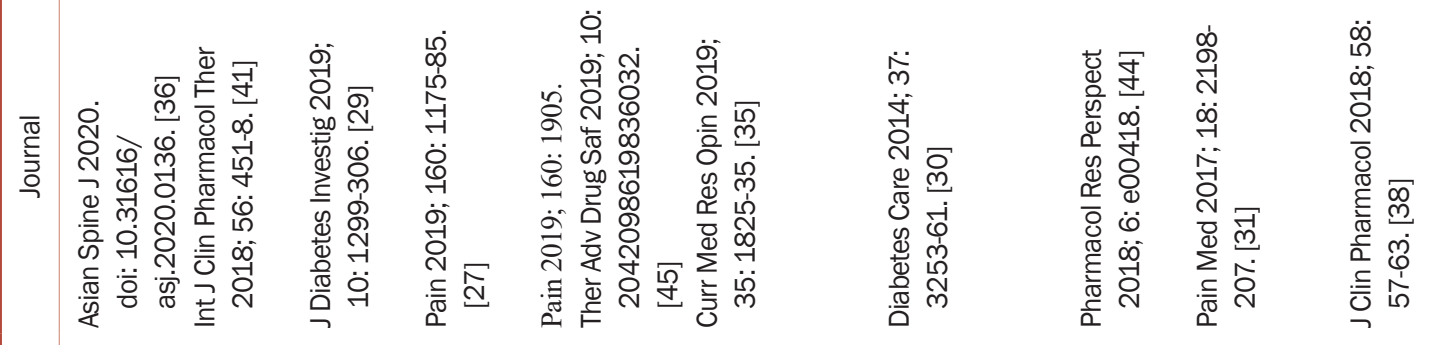

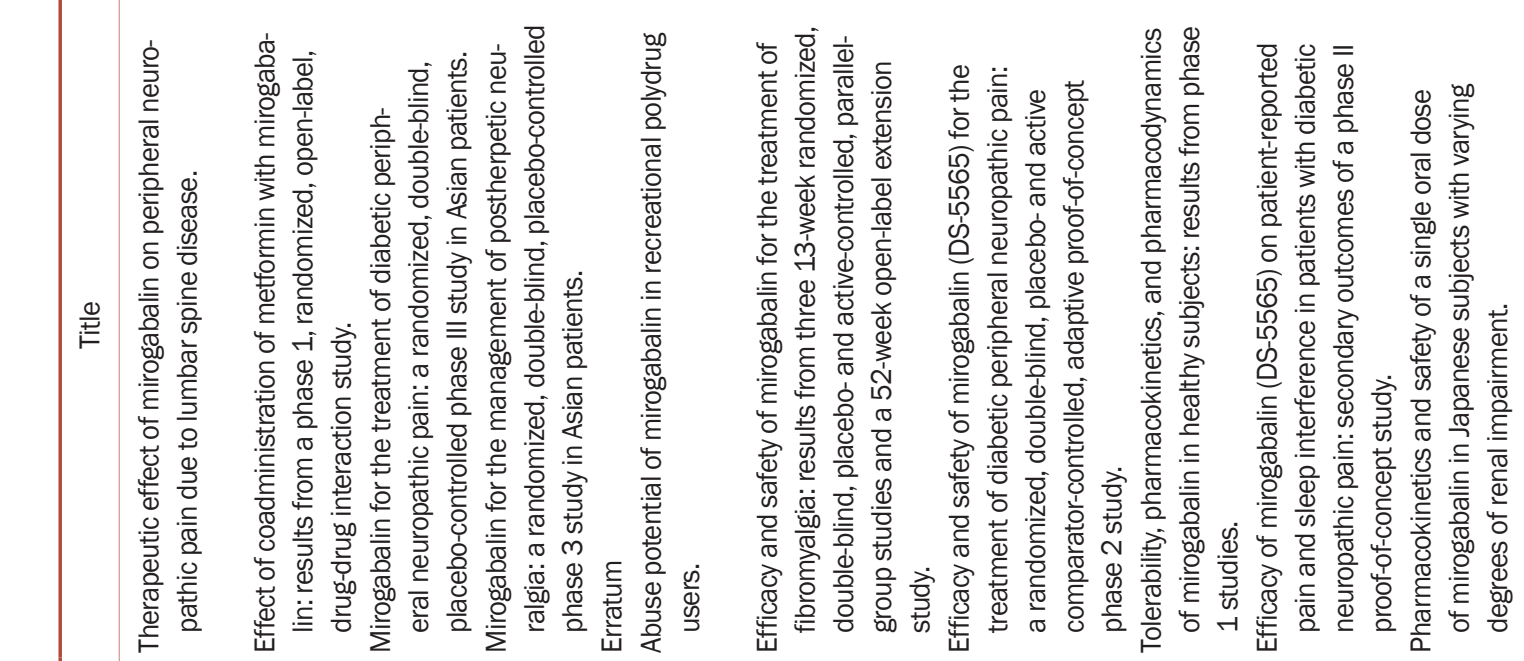

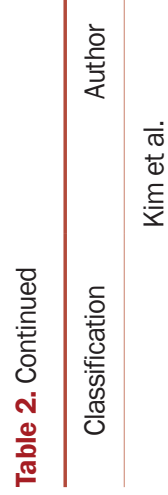

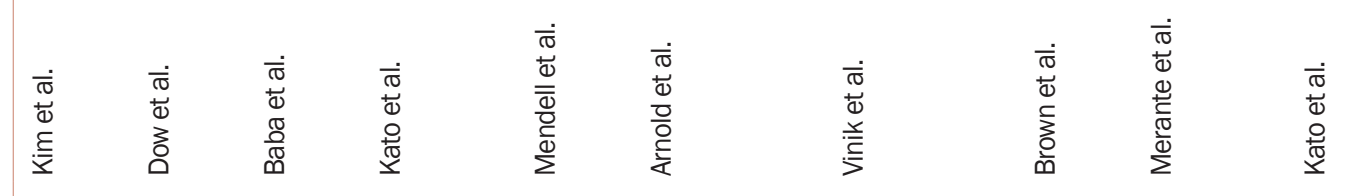




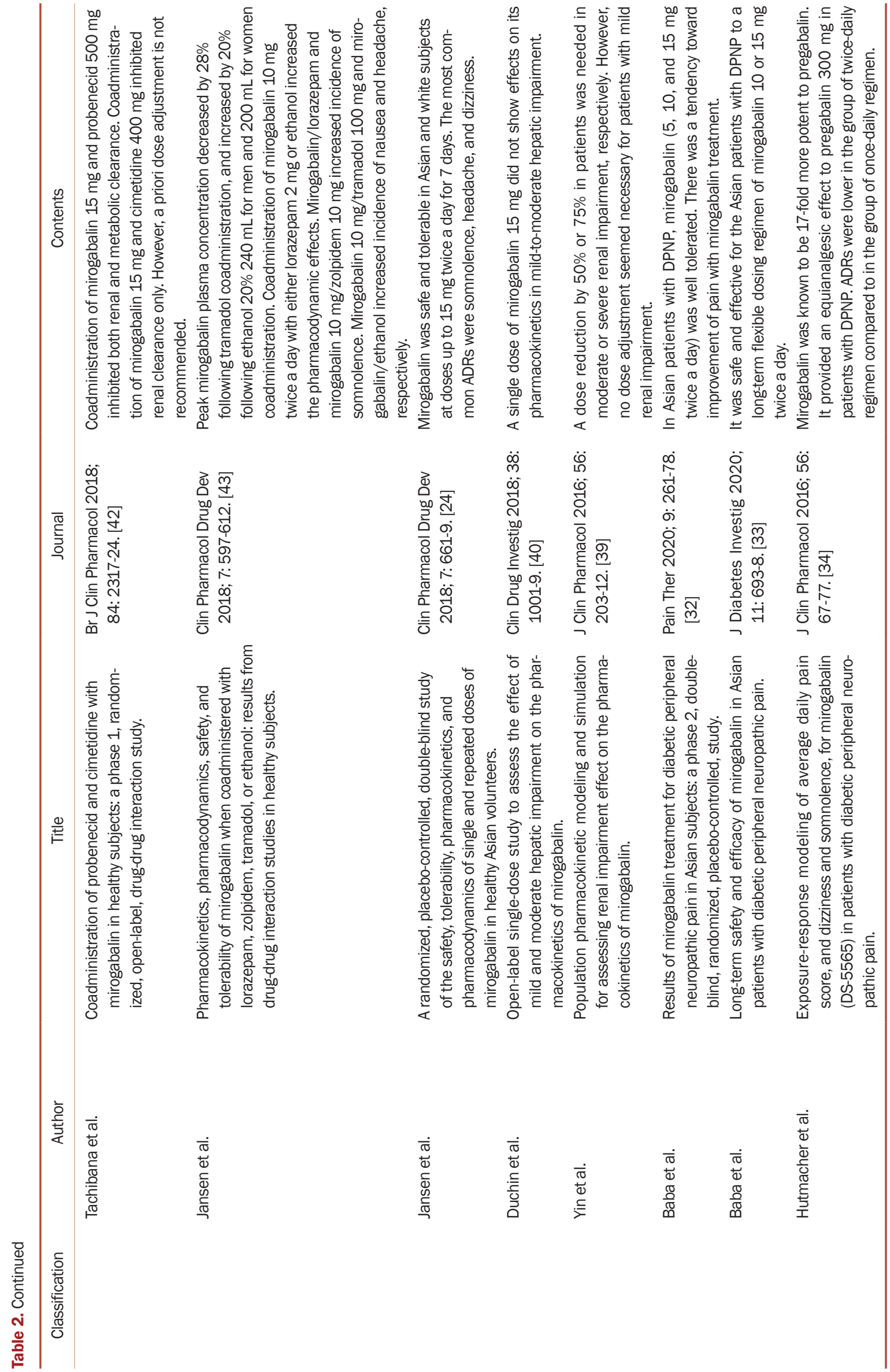




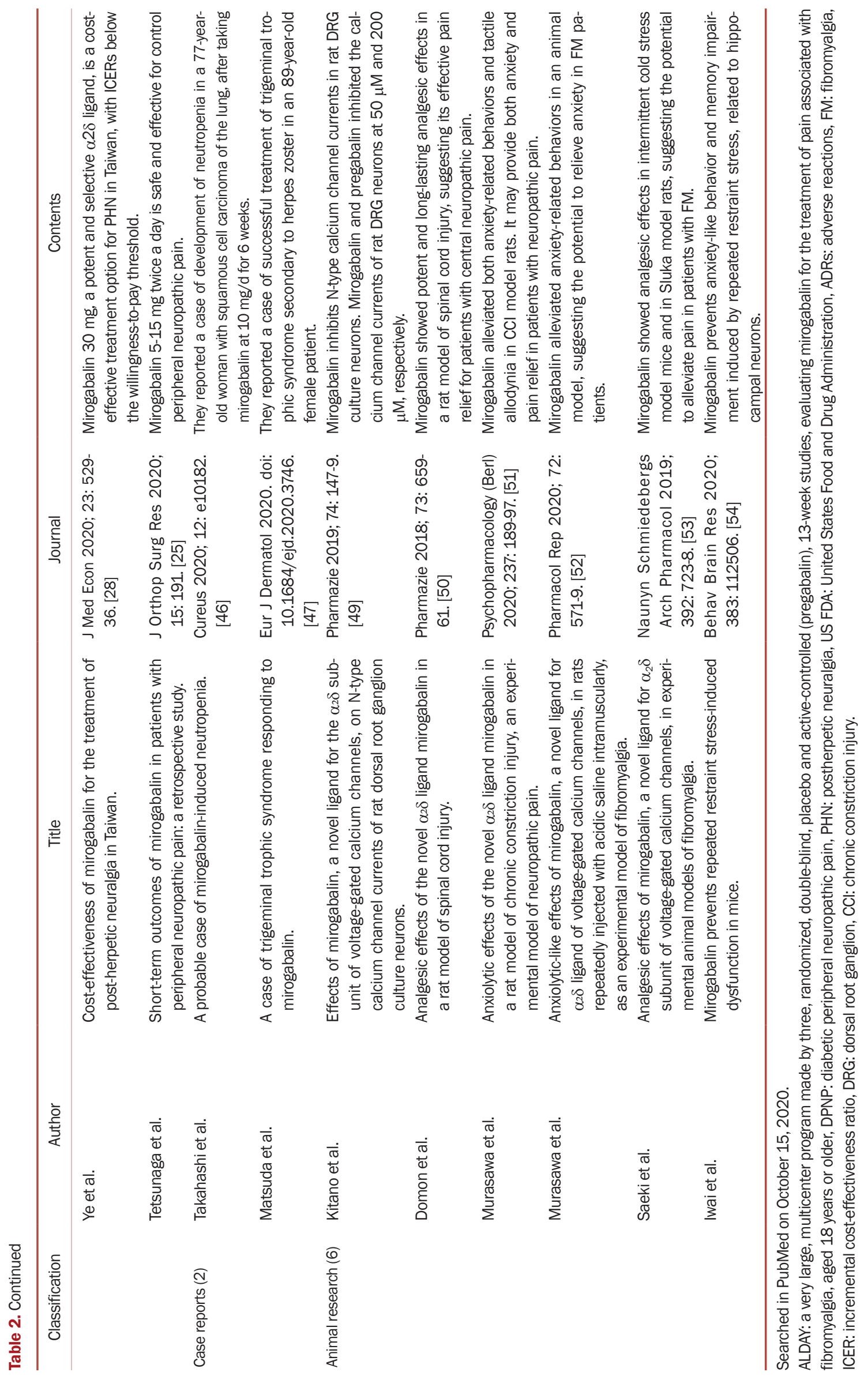




\section{Review articles, clinical trials, case reports, and animal studies}

Thirty-eight studies related to mirogabalin had been published prior to October 15, 2020 when searching PubMed. The mirogabalin studies include 8 review articles, 22 clinical trials, 2 case reports, and 6 animal studies (Table 2).

\section{1) Review articles}

From the 8 review articles, mirogabalin was suitable for the treatment of peripheral neuropathic pain, especially DPNP and PHN $[2,3,18]$. It is expected to become the 4 th United States Food and Drug Administration (U.S. FDA)approved drug for DPNP, along with pregabalin, duloxetine, and tapentadol [19]. It showed a superior result to pregabalin in the average daily pain score, with manageable ADRs (fatigue, dizziness, sedation, somnolence, ataxia, weight gain, and edema) for DPNP [20,21].

However, it showed a negative result with fibromyalgia [from the pain in patients with fibromyalgia (a very large, multicenter program made by three, randomized, doubleblind, placebo and active-controlled (pregabalin), 13week studies, evaluating mirogabalin for the treatment of pain associated with fibromyalgia, aged 18 years or older, ALDAY) study] [22].

Relatively clear mechanisms of action for mirogabalin, related to binding affinity and dissociation rates to the $\alpha_{2} \delta$ 1 and $\alpha_{2} \delta-2$ subunits of VGCCs, especially in the DRG and cerebellum have been introduced $[6,23]$.

\section{2) Clinical trials}

\section{(1) Heathy volunteers}

For healthy volunteers, mirogabalin, at doses up to $15 \mathrm{mg}$ twice a day for 7 days, showed safety and tolerability with ADRs [24].

\section{(2) Patients with PHN}

Mirogabalin was effective on various neuropathic pain syndromes [25]. In patients with PHN, 10 or $15 \mathrm{mg}$ of mirogabalin twice a day for 52 weeks showed effective control of pain, with mild to moderate ADRs (An Asian, phase 3, multicenter, randomized double-blind, placebocontrolled 14-week study of DS-5565 in patients with postherpetic neuralgia followed by a 52-week open-label extension, NEUCOURSE clinical trial) [26]. All doses (15, 20 , and $30 \mathrm{mg} / \mathrm{d}$ ) for 14 weeks were superior to a placebo for relieving PHN [27]. Mirogabalin $30 \mathrm{mg}$, after a 1-month cessation of pregabalin, also showed effectiveness and safety for 187 patients with PHN in Taiwan [28].

\section{(3) Patients with DPNP}

In patients with DPNP, mirogabalin $30 \mathrm{mg}$ showed significant pain relief, compared to a placebo, in a phase 3 study (an Asian, phase 3, multicenter, randomized, doubleblind, placebo-controlled 14-week study of mirogabalin in patients with diabetic peripheral neuropathic pain, followed by a 52 -week open-label extension, REDUCER clinical trial) in Japan, Taiwan, South Korea, and Malaysia [29]. In a phase 2 study, the average daily pain score was reduced at a daily dose of mirogabalin of $5,10,15,20$, and $30 \mathrm{mg}$ for 5 weeks, compared to a placebo [30]. A similarlydesigned phase 2 study showed a decreased average daily pain score and sleep interference score [31]. In another phase 2 study, mirogabalin $(5,10,15,20$, and $30 \mathrm{mg}$ twice a day) decreased the average daily pain score, average daily sleep-interference score, and ADRs [32]. In addition, mirogabalin 10 or $15 \mathrm{mg}$ twice a day showed long-term (52 wk) safety and efficacy in patients with DPNP [33].

Mirogabalin (17.7 mg) was estimated to be 17 -fold more potent than pregabalin $(300 \mathrm{mg}$ ) in patients with DPNP. Twice-daily dosing of mirogabalin decreased dizziness more than once-daily dosing [34].

\section{(4) Patients with fibromyalgia}

Unfortunately, mirogabalin showed a negative result. Both mirogabalin $15 \mathrm{mg}$ once and twice daily did not improve the worst weekly average daily pain score at week 13 [35]. In addition, the ALDAY phase 3 study did not decrease pain in patients with fibromyalgia [22].

\section{(5) Patients with neuropathic pain from lumbar spine} disease

Mirogabalin improved leg symptoms, low back pain, and sleep disturbance in patients with lumbar spine disease [36].

(6) Patients with neuropathic pain in renal or hepatic dysfunction

In patients with renal failure, a fixed dose of $7.5 \mathrm{mg}$ once and twice a day reduced DPNP and PHN with a tolerable level of ADRs [37]. In moderate or severe renal dysfunction, a dose reduction was needed by $50 \%$ or $70 \%$. However, a dose adjustment was not needed in mild renal dysfunction $[38,39]$.

In patients with mild or moderate hepatic impairment, a single $15 \mathrm{mg}$ dose of mirogabalin did not produce signifi- 
cant ADRs [40].

\section{(7) Coadministration with other drugs}

Metformin, a biguanide antihyperglycemic agent for type 2 diabetes, is an essential drug for control of blood glucose. However, the U.S. FDA recalled some types of metformin because it may contain N-nitrosodimethylamine (NMDA), a hepatotoxic and carcinogenic organic compound, above the acceptable intake limit on October 5, 2020. Even though the use of metformin is currently reduced, it is difficult to avoid coadministration. Coadministration of metformin and mirogabalin was well-tolerated in healthy volunteers [41].

Probenecid, treating gout and hyperuricemia, has known interactions with some commonly-used drugs, such as non-steroidal anti-inflammatory drugs (indomethacin, ketoprofen, ketorolac, and naproxen), antibiotics (cephalosporins, quinolones, and penicillins), methotrexate, acyclovir, and lorazepam, and reduces excretion of these drugs [42].

Probenecid inhibits both renal and metabolic clearance; cimetidine reduces renal clearance. Even though renal excretion of a single oral dose of mirogabalin $15 \mathrm{mg}$ in healthy volunteers was slightly decreased with coadministration of both probenecid $500 \mathrm{mg}$ and cimetidine 400 $\mathrm{mg}$, adjustment of the dose of mirogabalin was not recommended clinically in a phase 1 study [42].

Peak plasma mirogabalin concentration decreased by $28 \%$ following tramadol coadministration, but increased by $20 \%$ following ethanol coadministration. Coadministration with either lorazepam or ethanol increased pharmacodynamic parameters. In addition, mirogabalin/ lorazepam and mirogabalin/zolpidem increased sleepiness. Mirogabalin/tramadol and mirogabalin/ethanol increased incidence of nausea and headache, respectively [43].

(8) Bioavailability to food intake

Fed (high-fat meal) or fasting states in healthy volunteers did not affect bioavailability after taking a single dose of mirogabalin $15 \mathrm{mg}$. No food restriction was needed when taking mirogabalin in a phase 1 study [44].

\section{(9) Abuse potential}

At supra-therapeutic doses (over 4 times the therapeutic dose), mirogabalin showed higher abuse potential than a placebo, but lower abuse potential than diazepam and pregabalin [45].

\section{3) Case reports}

There have been 2 case reports. Neutropenia induced by mirogabalin $10 \mathrm{mg}$ for 6 weeks was noted in patients with squamous cell carcinoma of the lung, even after cessation of coadministration of acetaminophen and mexiletine [46].

There was a case of trigeminal trophic syndrome (TTS) responding to mirogabalin [47]. TTS is a rare facial ulceration which may appear after damage to the trigeminal nerve or its central sensory connections (herpes zoster or Hansen's disease). A triad of TTS consists of anesthesia, paresthesia, and facial ulceration [48]. It is not uncommon to use mirogabalin for the treatment of herpes zoster in the territory of the trigeminal nerve.

\section{4) Animal research}

Six animal studies can be found in PubMed. Focused on the unique binding ability of mirogabalin and pregabalin to the $\alpha_{2} \delta$-1 subunit of VGCCs, on N-type calcium channel currents of rat DRG culture neurons, they inhibit N-type calcium currents at $50 \mu \mathrm{M}$ and $200 \mu \mathrm{M}$, respectively. The authors concluded that the potent and prolonged analgesic effects of mirogabalin are associated with its potent and selective binding to the $\alpha_{2} \delta$-1 subunits, resulting in functional inhibition of calcium channel currents [49]. However, it is better to understand the mechanisms of action for mirogabalin as resulting from slow dissociation from the $\alpha_{2} \delta$-1 subunits in the DRGs and rapid dissociation from the $\alpha_{2} \delta$-2 subunits in the cerebellum (rather than its more potent and selective binding affinity to the $\alpha_{2} \delta$-1 subunits in comparison to the $\alpha_{2} \delta$-2 subunits) from their previous article [6].

In a spinal cord injury model, established by acute compression of the spinal cord at the T6-T7 level in rats, mirogabalin showed potent and long-lasting central analgesic effects [50].

In a chronic constriction injury (CCI) model in rats, mirogabalin showed dose-dependent anxiety-related behavior, as well as tactile allodynia [51]. A similar study on the anxiolytic effect of mirogabalin, by the same study group, was performed in the Sluka model (2 intramuscular injections of acid saline into the gastrocnemius muscle). A mirogabalin study showed an analgesic effect in an intermittent cold stress model in mice and Sluka model rats, suggesting effectiveness in patients with fibromyalgia [52]. Mirogabalin alleviated anxiolytic behavior in Sluka model rats, suggesting potential to relieve anxiety in fibromyalgia [53]. On the other hand, there was no analgesic effect in patients with fibromyalgia in the ALDAY phase 3 program and a 13-week study using mirogabalin $15 \mathrm{mg}$ once or twice a day [22]. Another anxiety-related study showed 
that mirogabalin protected multiple brain functions from repeated restraint stress ( $2 \mathrm{hr} /$ day), mediated by inhibition of hippocampal neuron hyperactivation [54].

\section{Clinical application of mirogabalin}

\section{1) Pharmacokinetics}

Available mirogabalin besylate tablets include 2.5, 5, 10, and $15 \mathrm{mg}$. The initial dose for adults without renal dysfunction is recommended starting from $5 \mathrm{mg}$, given orally twice a day. The dose is gradually increased by $5 \mathrm{mg}$, at an interval of at least a week, to $15 \mathrm{mg}$ at the $3 \mathrm{rd}$ week $[2,3,25$ 33].

After taking oral mirogabalin, maximum plasma concentration was achieved at 1 hour [19,39]. Its plasma protein binding was relatively low, at less than $25 \%$ [39]. It was mostly excreted by the kidney, and underwent minimal metabolism [19].

According to renal function (creatinine clearance, $\mathrm{Cl}_{\mathrm{cr}}$ ), the administration dose of mirogabalin should be adjusted in at least 3 stages: (1) a mild degree $\left(90>\mathrm{Cl}_{\mathrm{cr}} \geq 60\right.$ $\mathrm{mL} / \mathrm{m})$, (2) a moderate degree $\left(60>\mathrm{Cl}_{\mathrm{cr}} \geq 30 \mathrm{~mL} / \mathrm{m}\right)$, and (3) a severe degree $\left(30>\mathrm{Cl}_{\mathrm{cr}} \mathrm{mL} / \mathrm{m}\right)$ of renal dysfunction. In mild renal dysfunction, the initial dose starts from 5 $\mathrm{mg}$ twice a day, slowly increased by $5 \mathrm{mg}$ at an interval of 1 week, to $15 \mathrm{mg}$. In moderate renal dysfunction, the initial dose starts from $2.5 \mathrm{mg}$ twice a day, slowly increased by 2.5 $\mathrm{mg}$ at an interval of 1 week, to $7.5 \mathrm{mg}$ twice a day. In severe renal dysfunction, the initial dose starts from $2.5 \mathrm{mg}$ once a day, slowly increased by $2.5 \mathrm{mg}$ at an interval of 1 week, to $7.5 \mathrm{mg}$ once a day (Table 3) [55].

A dose reduction of mirogabalin is suggested by $50 \%$ and $75 \%$ in patients with moderate and severe renal dysfunction, respectively $[19,38]$. A single dose of mirogabalin 5 $\mathrm{mg}$ was tolerable in patients with various degree of renal failure, however, a dose adjustment should be considered in patients with severe degree and end-stage renal failure [38].

In a mild or moderate hepatic dysfunction, a single dose of mirogabalin $15 \mathrm{mg}$ was also tolerable [40].

\section{2) Pharmacodynamics}

Mirogabalin binds to and dissociates from the $\alpha_{2} \delta$ subunits. Its binding ability is stronger for $\alpha_{2} \delta-1$ than for $\alpha_{2} \delta-2$ subunits. It also dissociates from the $\alpha_{2} \delta$-1 subunits more slowly than the $\alpha_{2} \delta-2$ subunits [6]. Therefore, it showed a potent and long-lasting binding to the $\alpha_{2} \delta-1$ subunits, which exhibited a therapeutic analgesic effect through the DRGs, and a low incidence of ADRs through the cerebellum due to weak and shorting-lasting binding to the $\alpha_{2} \delta$ 2 subunits, with a wide safety index (a broad therapeutic window).

In a streptozotocin-induced diabetic model in rats, an effective analgesic dose $\left(\mathrm{ED}_{50}\right)$ of mirogabalin was 4 times lower than that of pregabalin. The effective dose for central nervous system ADRs $\left(\mathrm{ED}_{50}\right)$ occurs at about double the analgesic dose $\left(\mathrm{ED}_{50}\right)$ in the Rotarod performance test, and at 10 times the analgesic dose $\left(\mathrm{ED}_{50}\right)$ in a locomotor activity test. The safety index (effective ADR dose/effective analgesic dose) of mirogabalin is 2.1 and 10.0 , compared to 0.4 and 4.2 for pregabalin, in the Rotarod performance and the locomotor activity test, respectively [6].

The most common ADRs in the 277 patients with DPNP developed in the nervous system $(28.0 \%)$, such as dizziness (9.4\%), somnolence $(6.1 \%)$, headache $(6.1 \%)$, and balance disorder (0.5\%). Gastrointestinal ADRs (13.7\%) included constipation (4.3\%), nausea (4.0\%), diarrhea $(2.9 \%)$, and vomiting (2.9\%). Generalized ADRs (13\%) included peripheral edema (4.7\%), fatigue (3.6\%), weight gain (1.8\%), urinary tract infection $(2.9 \%)$, hyperglycemia $(2.2 \%)$, and fall (1.4\%) [30].

Table 3. Initial administration, dose titration, and final maintenance dose for mirogabalin in renal failure

\begin{tabular}{|c|c|c|c|c|}
\hline \multirow{2}{*}{\multicolumn{2}{|c|}{ Stage of renal dysfunction }} & \multicolumn{3}{|c|}{ Renal dysfunction } \\
\hline & & \multirow{2}{*}{$\begin{array}{c}\text { Mild degree } \\
\left(90>\mathrm{Cl}_{\mathrm{cr}} \geq 60 \mathrm{~mL} / \mathrm{m}\right) \\
5 \mathrm{mg} \text { twice a day }\end{array}$} & \multirow{2}{*}{$\begin{array}{c}\text { Moderate degree } \\
\left(60>\mathrm{Cl}_{\mathrm{cr}} \geq 30 \mathrm{~mL} / \mathrm{m}\right) \\
2.5 \mathrm{mg} \text { twice a day }\end{array}$} & \multirow{2}{*}{$\begin{array}{c}\text { Severe degree } \\
\left(30>\mathrm{Cl}_{\mathrm{cr}} \mathrm{mL} / \mathrm{m}\right) \\
2.5 \mathrm{mg} \text { once a day }\end{array}$} \\
\hline Initial dosage & & & & \\
\hline Daily dose & & $10-30 \mathrm{mg}$ & $5-15 \mathrm{mg}$ & $2.5-7.5 \mathrm{mg}$ \\
\hline Effective dose & Minimal dose & 10 mg twice a day & 5 mg twice a day & 5 mg once a day \\
\hline & Recommended dose & 15 mg twice a day & 7.5 mg twice a day & $7.5 \mathrm{mg}$ once a day \\
\hline
\end{tabular}

Available mirogabalin besylate tablets include $2.5,5,10$, and $15 \mathrm{mg}$.

The initial dose for adults without renal dysfunction is recommended starting from $5 \mathrm{mg}$, given orally twice a day. The dose is gradually increased by 5 $\mathrm{mg}$, at an interval of at least a week, to $15 \mathrm{mg}$ twice a day at the 3rd week [2,3,25-33].

Adapted from Kitano et al. [Pharmacological, pharmacodynamics, and clinical profile of mirogabalin besylate (Tarlige ${ }^{\circledR}$ tablets $2.5 \mathrm{mg} \cdot 5 \mathrm{mg} \cdot 10 \mathrm{mg} \cdot 15$ mg)]. Nihon Yakurigaku Zasshi 2019; 154: 352-61. Japanese [55]. 


\section{3) Equianalgesic dose}

Mirogabalin $30 \mathrm{mg}$ showed similar pain relief, number needed to treat (NNT), and a slightly lower incidence of withdrawal ADRs, compared with pregabalin $600 \mathrm{mg}$, gabapentin over 1,200 mg, and duloxetine $60 \mathrm{mg}$ [19].

\section{CONCLUSIONS}

Maladaptation and dysregulation of the $\alpha_{2} \delta-1$ subunits of VGCCs may cause neuropathic pain [56]. Mirogabalin has a strong binding affinity to and slow dissociation property from $\alpha_{2} \delta-1$ in the DRGs of the spinal cord, exhibiting a potent and prolonged analgesic therapeutic effect. It also has an weak binding affinity to and fast dissociation property from $\alpha_{2} \delta-2$ in the Purkinje fibers of the cerebellum, exhibiting weak and short-lasting ADRs.

The starting dose is $5 \mathrm{mg}$ twice a day in the lst week, escalating to $10 \mathrm{mg}$ in the $2 \mathrm{nd}$ week, and finally achieving a dose of $15 \mathrm{mg}$ in the 3rd week. Dose adjustment is needed in patients with renal dysfunction. An equianalgesic daily dose for gabapentin over 1,200 mg or pregabalin $600 \mathrm{mg}$ is mirogabalin $30 \mathrm{mg}$. Food restriction is not needed. Tramadol decreases a peak concentration of mirogabalin, while ethanol increases it. Lorazepam or ethanol increases the pharmacodynamic effects of mirogabalin. Coadministration of lorazepam or zolpidem increases somnolence. Tramadol increases nausea and ethanol increases headache in patients taking mirogabalin.

A progression from gabapentin to pregabalin originated from its rapid peak in blood concentration ( $3 \mathrm{hr}$ to $1 \mathrm{hr}$ ) resulting from an increased absorption area expanding from the small intestine into the ascending colon, resulting in linear absorption [57]. Even though it is indicated for PHN and DPNP currently, it is also available for all types of central and peripheral neuropathic pain.

\section{CONFLICT OF INTEREST}

No potential conflict of interest relevant to this article was reported.

\section{FUNDING}

This study was supported by a 2-year research grant from Pusan National University (2019-2021).

\section{ORCID}

Jae-Yeon Kim, https://orcid.org/0000-0002-6314-8710

Salahadin Abdi, https://orcid.org/0000-0002-3892-9208

Billy Huh, https://orcid.org/0000-0002-2825-9474

Kyung-Hoon Kim, https://orcid.org/0000-0003-3925-8917

\section{REFERENCES}

1. Dolphin AC. The $\alpha 2 \delta$ subunits of voltage-gated calcium channels. Biochim Biophys Acta 2013; 1828: 1541-9.

2. Deeks ED. Mirogabalin: first global approval. Drugs 2019; 79: 463-8.

3. Deeks ED. Correction to: mirogabalin: first global approval. Drugs 2019; 79: 469.

4. Kim KH, Seo HJ, Abdi S, Huh B. All about pain pharmacology: what pain physicians should know. Korean J Pain 2020; 33: 108-20.

5. Hauser AS, Attwood MM, Rask-Andersen M, Schiöth HB, Gloriam DE. Trends in GPCR drug discovery: new agents, targets and indications. Nat Rev Drug Discov 2017; 16: 82942.

6. Domon Y, Arakawa N, Inoue T, Matsuda F, Takahashi M, Yamamura N, et al. Binding characteristics and analgesic effects of mirogabalin, a novel ligand for the $\alpha_{2} \delta$ subunit of voltage-gated calcium channels. J Pharmacol Exp Ther 2018; 365: 573-82.

7. Dolphin AC. Voltage-gated calcium channel $\alpha_{2} \delta$ subunits: an assessment of proposed novel roles. F1000Res 2018; 7: F1000 Faculty Rev-1830.

8. Dolphin AC. Calcium channel auxiliary $\alpha_{2} \delta$ and $\beta$ subunits: trafficking and one step beyond. Nat Rev Neurosci 2012; 13: 542-55.

9. Burnashev N. Calcium permeability of ligand-gated channels. Cell Calcium 1998; 24: 325-32.

10. Catterall WA. Voltage-gated calcium channels. Cold Spring Harb Perspect Biol 2011; 3: a003947.

11. D'Arco M, Margas W, Cassidy JS, Dolphin AC. The upregulation of $\alpha 2 \delta$-1 subunit modulates activity-dependent $\mathrm{Ca} 2+$ signals in sensory neurons. J Neurosci 2015; 35: 5891-903.

12. Hoppa MB, Lana B, Margas W, Dolphin AC, Ryan TA. $\alpha 2 \delta$ expression sets presynaptic calcium channel abundance and release probability. Nature 2012; 486: 122-5.

13. Davies A, Hendrich J, Van Minh AT, Wratten J, Douglas L, Dolphin AC. Functional biology of the alpha(2)delta subunits of voltage-gated calcium channels. Trends Pharmacol Sci 2007; 28: 220-8.

14. Chincholkar M. Analgesic mechanisms of gabapentinoids and effects in experimental pain models: a narrative review. Br J Anaesth 2018; 120: 1315-34.

15. Patel R, Dickenson AH. Mechanisms of the gabapentinoids 
and $\alpha 2 \delta$-1 calcium channel subunit in neuropathic pain. Pharmacol Res Perspect 2016; 4: e00205.

16. El-Awaad E, Pryymachuk G, Fried C, Matthes J, Isensee J, Hucho T, et al. Direct, gabapentin-insensitive interaction of a soluble form of the calcium channel subunit $\alpha_{2} \delta-1$ with thrombospondin-4. Sci Rep 2019; 9: 16272.

17. Brini M, Calì T, Ottolini D, Carafoli E. Neuronal calcium signaling: function and dysfunction. Cell Mol Life Sci 2014; 71: 2787-814.

18. Alcántara Montero A, Sánchez Carnerero CI, Goicoechea García C. Emerging therapies in clinical development and new contributions for neuropathic pain. Rev Esp Anestesiol Reanim 2019; 66: 324-34.

19. Javed S, Alam U, Malik RA. Mirogabalin and emerging therapies for diabetic neuropathy. J Pain Res 2018; 11: 1559-66.

20. Alyoubi RA, Alshareef AA, Aldughaither SM, Aljaroudi AM, Alabdulwahed A, Alduraibi FM, et al. Efficacy and safety of mirogabalin treatment in patients with diabetic peripheral neuropathic pain: a systematic review and meta-analysis of randomised controlled trials. Int J Clin Pract 2020. doi: 10.1111/ijcp.13744.

21. Calandre EP, Rico-Villademoros F, Slim M. Alpha ${ }_{2}$ delta ligands, gabapentin, pregabalin and mirogabalin: a review of their clinical pharmacology and therapeutic use. Expert Rev Neurother 2016; 16: 1263-77.

22. Merante D. The mirogabalin ALDAY phase 3 program in pain associated with fibromyalgia: the lessons learned. Curr Med Res Opin 2020; 36: 661-6.

23. Burgess J, Javed S, Frank B, Malik RA, Alam U. Mirogabalin besylate in the treatment of neuropathic pain. Drugs Today (Barc) 2020; 56: 135-49.

24. Jansen M, Warrington S, Dishy V, Ohwada S, Johnson L, Brown $\mathrm{K}$, et al. A randomized, placebo-controlled, doubleblind study of the safety, tolerability, pharmacokinetics, and pharmacodynamics of single and repeated doses of mirogabalin in healthy Asian volunteers. Clin Pharmacol Drug Dev 2018; 7: 661-9.

25. Tetsunaga $\mathrm{T}$, Tetsunaga $\mathrm{T}$, Nishida $\mathrm{K}$, Misawa $\mathrm{H}$, Takigawa $\mathrm{T}$, Yamane $\mathrm{K}$, et al. Short-term outcomes of mirogabalin in patients with peripheral neuropathic pain: a retrospective study. J Orthop Surg Res 2020; 15: 191.

26. Kato J, Matsui N, Kakehi Y, Murayama E, Ohwada S. Longterm safety and efficacy of mirogabalin in Asian patients with postherpetic neuralgia: results from an open-label extension of a multicenter randomized, double-blind, placebocontrolled trial. Medicine (Baltimore) 2020; 99: e21976.

27. Kato J, Matsui N, Kakehi Y, Murayama E, Ohwada S, Sugihara M. Mirogabalin for the management of postherpetic neuralgia: a randomized, double-blind, placebo-controlled phase 3 study in Asian patients. Pain 2019; 160: 1175-85.

28. Ye X, Gray E, Wang YF, Wang SJ. Cost-effectiveness of mirogabalin for the treatment of post-herpetic neuralgia in Tai- wan. J Med Econ 2020; 23: 529-36.

29. Baba M, Matsui N, Kuroha M, Wasaki Y, Ohwada S. Mirogabalin for the treatment of diabetic peripheral neuropathic pain: a randomized, double-blind, placebo-controlled phase III study in Asian patients. J Diabetes Investig 2019; 10: 1299306.

30. Vinik A, Rosenstock J, Sharma U, Feins K, Hsu C, Merante D; DS5565-A-U201 US Phase II Study Investigators. Efficacy and safety of mirogabalin (DS-5565) for the treatment of diabetic peripheral neuropathic pain: a randomized, double-blind, placebo- and active comparator-controlled, adaptive proofof-concept phase 2 study. Diabetes Care 2014; 37: 3253-61.

31. Merante D, Rosenstock J, Sharma U, Feins K, Hsu C, Vinik A; DS-5565-A-U201 US Phase 2 Study Investigators. Efficacy of mirogabalin (DS-5565) on patient-reported pain and sleep interference in patients with diabetic neuropathic pain: secondary outcomes of a phase II proof-of-concept study. Pain Med 2017; 18: 2198-207.

32. Baba M, Kuroha M, Ohwada S, Murayama E, Matsui N. Results of mirogabalin treatment for diabetic peripheral neuropathic pain in Asian subjects: a phase 2, double-blind, randomized, placebo-controlled, study. Pain Ther 2020; 9: 261-78.

33. Baba M, Matsui N, Kuroha M, Wasaki Y, Ohwada S. Longterm safety and efficacy of mirogabalin in Asian patients with diabetic peripheral neuropathic pain. J Diabetes Investig 2020; 11: 693-8.

34. Hutmacher MM, Frame B, Miller R, Truitt K, Merante D. Exposure-response modeling of average daily pain score, and dizziness and somnolence, for mirogabalin (DS-5565) in patients with diabetic peripheral neuropathic pain. J Clin Pharmacol 2016; 56: 67-77.

35. Arnold LM, Whitaker S, Hsu C, Jacobs D, Merante D. Efficacy and safety of mirogabalin for the treatment of fibromyalgia: results from three 13-week randomized, double-blind, placebo- and active-controlled, parallel-group studies and a 52week open-label extension study. Curr Med Res Opin 2019; 35: 1825-35.

36. Kim K, Isu T, Kokubo R, Iwamoto N, Morimoto D, Kawauchi $\mathrm{M}$, et al. Therapeutic effect of mirogabalin on peripheral neuropathic pain due to lumbar spine disease. Asian Spine J 2020. doi: 10.31616/asj.2020.0136.

37. Baba M, Takatsuna H, Matsui N, Ohwada S. Mirogabalin in Japanese patients with renal impairment and pain associated with diabetic peripheral neuropathy or post-herpetic neuralgia: a phase III, open-label, 14-week study. J Pain Res 2020; 13: 1811-21.

38. Kato M, Tajima N, Shimizu T, Sugihara M, Furihata K, Harada K, et al. Pharmacokinetics and safety of a single oral dose of mirogabalin in Japanese subjects with varying degrees of renal impairment. J Clin Pharmacol 2018; 58: 57-63.

39. Yin OQ, Merante D, Truitt K, Miller R. Population pharmaco- 
kinetic modeling and simulation for assessing renal impairment effect on the pharmacokinetics of mirogabalin. J Clin Pharmacol 2016; 56: 203-12.

40. Duchin K, Senaldi G, Warren V, Marbury T, Lasseter K, Zahir H. Open-label single-dose study to assess the effect of mild and moderate hepatic impairment on the pharmacokinetics of mirogabalin. Clin Drug Investig 2018; 38: 1001-9.

41. Dow J, Currie A, He L, Zaidi F, Zahir H. Effect of coadministration of metformin with mirogabalin: results from a phase 1 , randomized, open-label, drug-drug interaction study. Int J Clin Pharmacol Ther 2018; 56: 451-8.

42. Tachibana M, Yamamura N, Atiee GJ, Hsu C, Warren V, He L, et al. Coadministration of probenecid and cimetidine with mirogabalin in healthy subjects: a phase 1, randomized, open-label, drug-drug interaction study. Br J Clin Pharmacol 2018; 84: 2317-24.

43. Jansen M, Mendell J, Currie A, Dow J, He L, Merante D, et al. Pharmacokinetics, pharmacodynamics, safety, and tolerability of mirogabalin when coadministered with lorazepam, zolpidem, tramadol, or ethanol: results from drug-drug interaction studies in healthy subjects. Clin Pharmacol Drug Dev 2018; 7: 597-612.

44. Brown K, Mendell J, Ohwada S, Hsu C, He L, Warren V, et al. Tolerability, pharmacokinetics, and pharmacodynamics of mirogabalin in healthy subjects: results from phase 1 studies. Pharmacol Res Perspect 2018; 6: e00418.

45. Mendell J, Levy-Cooperman N, Sellers E, Vince B, Kelsh $\mathrm{D}$, Lee $\mathrm{J}$, et al. Abuse potential of mirogabalin in recreational polydrug users. Ther Adv Drug Saf 2019; 10: 2042098619836032.

46. Takahashi S, Ogata A, Nakamura M. A probable case of mirogabalin-induced neutropenia. Cureus 2020; 12: e10182.

47. Matsuda KM, Tanaka-Mizutsugu H, Kishi Y, Hino H, Kagami S. A case of trigeminal trophic syndrome responding to mirogabalin. Eur J Dermatol 2020. doi: 10.1684/ejd.2020.3746.

48. Kumar P, Thomas J. Trigeminal trophic syndrome. Indian J Dermatol 2014; 59: 75-6.

49. Kitano Y, Wakimoto S, Tamura S, Kubota K, Domon Y, Ara- kawa N, et al. Effects of mirogabalin, a novel ligand for the $\alpha_{2} \delta$ subunit of voltage-gated calcium channels, on N-type calcium channel currents of rat dorsal root ganglion culture neurons. Pharmazie 2019; 74: 147-9.

50. Domon Y, Kitano Y, Makino M. Analgesic effects of the novel $\alpha_{2} \delta$ ligand mirogabalin in a rat model of spinal cord injury. Pharmazie 2018; 73: 659-61.

51. Murasawa H, Kobayashi H, Saeki K, Kitano Y. Anxiolytic effects of the novel $\alpha_{2} \delta$ ligand mirogabalin in a rat model of chronic constriction injury, an experimental model of neuropathic pain. Psychopharmacology (Berl) 2020; 237: 189-97.

52. Murasawa H, Kobayashi H, Yasuda SI, Saeki K, Domon Y, Arakawa N, et al. Anxiolytic-like effects of mirogabalin, a novel ligand for $\alpha_{2} \delta$ ligand of voltage-gated calcium channels, in rats repeatedly injected with acidic saline intramuscularly, as an experimental model of fibromyalgia. Pharmacol Rep 2020; 72: 571-9.

53. Saeki K, Yasuda SI, Kato M, Kano M, Domon Y, Arakawa N, et al. Analgesic effects of mirogabalin, a novel ligand for $\alpha_{2} \delta$ subunit of voltage-gated calcium channels, in experimental animal models of fibromyalgia. Naunyn Schmiedebergs Arch Pharmacol 2019; 392: 723-8.

54. Iwai T, Kikuchi A, Oyama M, Watanabe S, Tanabe M. Mirogabalin prevents repeated restraint stress-induced dysfunction in mice. Behav Brain Res 2020; 383: 112506.

55. Kitano Y, Kai K, Yamamura N, Yoshiba S, Kuroha M. [Pharmacological, pharmacodynamics, and clinical profile of mirogabalin besylate (Tarlige ${ }^{\circledR}$ tablets $2.5 \mathrm{mg} \cdot 5 \mathrm{mg} \cdot 10 \mathrm{mg} \cdot 15$ mg)]. Nihon Yakurigaku Zasshi 2019; 154: 352-61. Japanese.

56. Gong N, Park J, Luo ZD. Injury-induced maladaptation and dysregulation of calcium channel $\alpha_{2} \delta$ subunit proteins and its contribution to neuropathic pain development. Br J Pharmacol 2018; 175: 2231-43.

57. Bockbrader HN, Wesche D, Miller R, Chapel S, Janiczek N, Burger P. A comparison of the pharmacokinetics and pharmacodynamics of pregabalin and gabapentin. Clin Pharmacokinet 2010; 49: 661-9. 